\title{
Exercício físico e artrite reumatoide: Possibilidades de intervenção de um protocolo
}

\section{de treinamento}

\author{
Physical exercise and rheumatoid arthritis: Possibilities of intervention of a training protocol \\ Ejercicio físico y artritis reumatoide: Posibilidades de intervención de un protocolo de \\ entrenamiento
}

Recebido: 20/07/2021 | Revisado: 23/07/2021 | Aceito: 27/07/2021 | Publicado: 03/08/2021

\author{
Ângelo Solano Negrão \\ ORCID: https://orcid.org/0000-0002-4713-4941 \\ Universidade Federal do Pará, Brasil \\ E-mail: angelosolano16@gmail.com \\ Igor Mateus Fernandes de Oliveira \\ ORCID: https://orcid.org/0000-0001-6285-7833 \\ Universidade do Estado do Pará, Brasil \\ E-mail: Igorfernandes500@ @otmail.com \\ Douglas Alencar Vieira \\ ORCID: https://orcid.org/0000-0002-5552-5037 \\ Universidade Federal de Sergipe, Brasil \\ E-mail: dougvieiira08@gmail.com \\ Anderson Bentes de Lima \\ ORCID: https://orcid.org/0000-0002-0534-2654 \\ Universidade do Estado do Pará, Brasil \\ E-mail: andersonbentes@uepa.br \\ Higson Rodrigues Coelho \\ ORCID: https://orcid.org/0000-0001-9585-2334 \\ Universidade do Estado do Pará, Brasil \\ E-mail: higson.coelho@uepa.br
}

\begin{abstract}
Resumo
$\mathrm{O}$ estudo analisa os efeitos de um protocolo de exercícios físicos em uma mulher com Artrite Reumatoide. Metodologicamente, configura-se como um estudo de caso de caráter quantitativo e qualitativo, sendo dividido em três etapas: 1-avaliação física de capacidade funcional, aplicação de questionários para investigação do estado da doença, qualidade de vida e depressão; 2-intervenção através da elaboração de um protocolo de treinamento; 3 reavaliação dos testes para obtenção de resultados comparativos. Os resultados obtidos demonstram uma evolução significativa nos aspectos da avaliação física e psicológica, já no aspecto da capacidade funcional o resultado foi pouco satisfatório. $\mathrm{Na}$ avaliação física, a sujeita apresentou resultados satisfatórios quanto à massa magra (33.40 $\pm 0.60, \Delta \%=2,5 \%)$, massa gorda $(9.35 \pm 0.93, \Delta \%=13.0 \%)$, percentual de gordura $(21.87 \pm 2.03, \Delta \%=12,3 \%)$, IMC $(18.87 \pm 0.37, \Delta \%=2,8 \%)$. Na análise da qualidade de vida obtivemos os resultados em cada domínio: no DF $(2.64 \pm 0.70, \Delta \%=31,8 \%)$, no DPS $(2.80 \pm 0.28, \Delta \%=13,3 \%)$, DS $(3.31 \pm 0.02, \Delta \%=0,9 \%)$ e no DA $(3.17 \pm 0.10$, $\Delta \%=4,8 \%)$. Em relação à capacidade funcional foram CF $(2.68 \pm 0.08, \Delta \% 4,7 \%)$, que demonstra pouca evolução em termos quantitativos, porém, tornam-se significantes pela melhora em suas atividades cotidianas. E por fim, no teste de depressão, houve um progresso tímido $(22.50 \pm 2.10, \Delta \%=12,5 \%)$. Conclui-se que os exercícios físicos têm uma grande importância no que se refere à artrite reumatoide da pessoa estudada, que se permite a aplicação de exercícios físicos como um meio não medicamentoso no tratamento da AR para a indivídua investigado se mostrou uma alternativa viável que revigora os índices e o cotidiano da estudada.
\end{abstract}

Palavras-chave: Exercício físico; Artrite reumatoide; Qualidade de vida.

\begin{abstract}
The study examines the effects of an exercise protocol in a woman with Rheumatoid Arthritis. Methodologically, it is a quantitative and qualitative case study, divided into three stages: 1-physical assessment of functional capacity, application of questionnaires to investigate the state of the disease, quality of life and depression; 2-intervention through the elaboration of a training protocol; 3-reassessment of tests to obtain comparative results. The results obtained demonstrate a significant evolution in the aspects of physical and psychological assessment, whereas in the aspect of functional capacity the result was unsatisfactory. In the physical evaluation, the subject presented satisfactory results in terms of lean mass $(33.40 \pm 0.60,=\%=2.5 \%)$, fat mass $(9.35 \pm 0.93, \Delta \%=13.0 \%)$, fat percentage $(21.87 \pm 2.03, \Delta \%=12.3 \%)$, BMI $(18.87 \pm 0.37, \Delta \%=2.8 \%)$. In the analysis of quality of life, we obtained the results in each domain: in the DF $(2.64 \pm 0.70, \Delta \%=31.8 \%)$, in the DPS $(2.80 \pm 0.28, \Delta \%=13.3 \%)$, DS $(3.31 \pm 0.02, \Delta \%=0.9 \%)$ and in the DA $(3.17 \pm 0.10, \Delta \%=4.8 \%)$. Regarding the functional capacity, they were FC $(2.68 \pm 0.08, \Delta \% 4.7 \%)$, which
\end{abstract}


shows little evolution in quantitative terms, however, they become significant due to the improvement in their daily activities. Finally, in the depression test, there was timid progress $(22.50 \pm 2.10, \Delta \%=12.5 \%)$. It is concluded that physical exercise is of great importance with regard to the studied person's rheumatoid arthritis, which allows the application of physical exercise as a non-pharmacological means in the treatment of RA for the investigated individual proved to be a viable alternative that invigorates the indexes and the daily life of the studied one.

Keywords: Physical exercise; Arthritis, Rheumatoid; Quality of life.

\section{Resumen}

El estudio examina los efectos de un protocolo de ejercicio en una mujer con artritis reumatoide. Metodológicamente, se trata de un estudio de caso cuantitativo y cualitativo, dividido en tres etapas: 1-evaluación física de la capacidad funcional, aplicación de cuestionarios para investigar el estado de la enfermedad, calidad de vida y depresión; 2 intervención mediante la elaboración de un protocolo de formación; 3-reevaluación de pruebas para obtener resultados comparativos. Los resultados obtenidos demuestran una evolución significativa en los aspectos de evaluación física y psicológica, mientras que en el aspecto de capacidad funcional el resultado fue insatisfactorio. En la evaluación física, el sujeto presentó resultados satisfactorios en cuanto a masa magra $(33,40 \pm 0,60,=\%=2,5 \%)$, masa grasa $(9,35 \pm$ $0,93, \Delta \%=13,0 \%)$, porcentaje de grasa $(21,87 \pm 2,03, \Delta \%=12,3 \%), \operatorname{IMC}(18,87 \pm 0,37, \Delta \%=2,8 \%)$. En el análisis de la calidad de vida, obtuvimos los resultados en cada dominio: en el DF $(2,64 \pm 0,70, \Delta \%=31,8 \%)$, en el DPS $(2,80$ $\pm 0,28, \Delta \%=13,3 \%)$, DS $(3,31 \pm 0,02, \Delta \%=0,9 \%)$ y en el DA $(3,17 \pm 0,10, \Delta \%=4,8 \%)$. En cuanto a la capacidad funcional, fueron $\mathrm{CF}(2,68 \pm 0,08, \Delta \% 4,7 \%)$, lo que muestra poca evolución en términos cuantitativos, sin embargo, se vuelven significativos debido a la mejora en sus actividades diarias. Finalmente, en la prueba de depresión, hubo un progreso tímido $(22,50 \pm 2,10, \Delta \%=12,5 \%)$. Se concluye que el ejercicio físico es de gran importancia con respecto a la artritis reumatoide de la persona estudiada, que permite la aplicación del ejercicio físico como medio no farmacológico en el tratamiento de la AR para el individuo investigado que resultó ser una alternativa viable que dinamiza el índices y la vida diaria del estudiado.

Palabras clave: Ejercicio físico; Artritis reumatoide; Calidad de vida.

\section{Introdução}

O presente estudo intenciona as possibilidades de intervenção das atividades físicas em pessoas com doenças reumatológicas. No mundo a Artrite Reumatoide (AR) é uma doença que atinge aproximadamente $0,5 \%$ a $1 \%$ da população mundial adulta, não sendo delimitada a grupos étnicos específicos. No Brasil, foi encontrado o predomínio de até $1 \%$ da população adulta, correspondendo a uma estimativa de 1.300.000 pessoas afetadas (Mota et al., 2011).

A Artrite reumatoide é uma enfermidade sistêmica autoimune, tendo como característica principal a apresentação de inflamação sinovial, lesão cartilaginosa e deformação articular; gera ainda aparições sistêmicas e associa-se a múltiplas doenças (Mota, Laurindo, \& Santos Neto, 2010a). Para Sociedade Brasileira de Reumatologia (SBR), AR é uma doença inflamatória crônica que pode afetar várias articulações. O motivo é desconhecido e atinge principalmente as mulheres, até duas vezes mais que os homens. Geralmente, a AR tem início com a entrada na fase adulta (entre 30 e 40 anos) e tende a incidir com o aumento da idade (Sociedade Brasileira de Reumatologia, 2017).

Porém, a enfermidade não está presente apenas em pessoas adultas. De acordo com alguns autores afirmam em seu estudo que o sedentarismo em crianças é responsável pelo aumento pandêmico da obesidade infantil, e que esta inatividade física ainda pode acarretar em outras doenças coronárias, como cardiovasculares, endócrinas e osteoarticulares (Gualano et al., 2011).

Levando em consideração este fato, uma publicação na Revista Brasileira de Atividade Física e Saúde, no ano de 2009, relata um grande aumento de estudos com a finalidade de averiguar os resultados dos exercícios físicos (EF) no processo de tratamento com pacientes apresentando $\mathrm{AR}$, estes exercícios são indispensáveis, pois, a prática regular aumenta a mobilidade articular, a funcionalidade do indivíduo e até mesmo o humor, sem malefícios articulares significativos ou agrave no processo inflamatório (Külkamp, Dario, Gevaerd, \& Domenech, 2012).

Outro fator a elencar é que pessoas com AR exibem um prevalecimento, maior que a média presente na população de modo geral, de alterações de quadros depressivos e de ansiedade que varia de $13 \%$ a $47 \%$ (Costa, Brasil, Papi, \& Azevedo, 2008). Não existe um consenso literário que defina um maior surgimento desses quadros na AR, contudo vale destacar que 
essas alterações de humor aumentam as reclamações dos pacientes, tornando dificultoso a continuidade no atendimento, e algumas vezes agravando o quadro de evolução da doença (Cavanaugh, Furlanetto, Creech, \& Powell, 2001).

O diagnóstico do quadro da doença depende da ligação de vários sintomas e amostras clínicas, exames laboratoriais e radiográficos; dentre elas estão: rigidez matinal, artrite em três ou mais articulações, artrite nas articulações da mão, artrite simétrica, nódulos reumatoides, alterações radiográficas, fator reumatoide sérico positivo (Laurindo et al., 2002). Autores defendem que a gravidade da doença é dividida em: leve, moderada e grave (Louzada-Junior, Souza, Toledo, \& Ciconelli, 2007).

Foi efetuado um monitoramento de gastos do Ministério da Saúde com medicamentos para o tratamento da AR, este estudo levantou os valores custeados entre os anos de 2010 a 2014, e chegou à somatória de 1,03 Bilhões de reais no orçamento, causando um consumo médio de 5,76\% nos recursos do Componente Especializado da Assistência Farmacêutica (Oliveira, 2015). Estes dados apontam a necessidade de instrumentos que auxiliem profissionais da saúde com os tratamentos não farmacológicos, contribuindo para a qualidade de vida dos pacientes acometidos com AR.

O EF apresenta-se como uma alternativa para reduzir o número e o tempo de internações hospitalares, os sintomas da AR e seu avanço, bem como as ações inflamatórias que causam dor e limitam o movimento. Além de contribuir para melhoria do sistema cardiovascular e músculo esquelético; reduz também problemas relacionados ao psicológico e emocional, assim como os custos para o cuidado à saúde (Rosa et al., 2018). Além disso, são acessíveis à população e podem ser adaptados para diferentes casos clínicos, levando em consideração a individualidade biológica de cada pessoa.

Com isso, este estudo se justifica na tentativa de elaborar um protocolo de treinamento físico com a finalidade de melhorar a qualidade de vida de uma mulher adulta com 49 anos de idade, que apresenta um estado grave de AR, visando aprimorar sua capacidade funcional, diminuir a incidência de dor, força muscular, mostrar uma alternativa para diminuir os gastos públicos utilizados no tratamento da doença, assim como contribuir para elaboração de treinamentos físicos que possam ser utilizados posteriormente por outros profissionais da saúde. O interesse por este tema surgiu a partir da vivência em estágios dentro de academias e estúdio de Pilates, obtendo uma breve experiência prática com alunos acometidos pela doença, por intermédio do EF.

O estudo objetiva analisar os efeitos da aplicação de um protocolo de EF em uma pessoa acometida com AR grave. E específicos: planejar e implementar um protocolo de treinamento físico adaptado para pessoas com AR; comparar os impactos, antes e pós, das capacidades físicas e autonomia funcional, tais como: mobilidade articular, força, equilíbrio e percentual de gordura, de uma pessoa com AR submetida ao protocolo de EF de 03 meses; verificar os efeitos do desenvolvimento do protocolo de EF sobre o quadro depressivo, do caso em estudo de uma pessoa acometida com AR.

\section{Exercício Físico e Artrite Reumatóide}

Com o propósito de identificar as principais linhas de estudos pesquisados no que diz respeito a EF como ferramenta de auxilio no tratamento da AR, este tópico tomará por base a contribuição de alguns autores que fizeram resgates históricos ou pesquisas literárias que possam fornecer informações para o melhor entendimento em relação aos benefícios apresentados.

Ainda hoje não é possível traçar um método ou protocolo padrão de EF no tratamento da AR, entretanto, diversas recomendações são apresentadas na literatura, que apesar de divergirem em relação aos exercícios, frequência, duração e intensidade, acabam convergindo na afirmação de que os EF são seguros para os pacientes e eficazes no tratamento da doença. E ainda pode ser verificado que, na maioria dos estudos analisados, houve melhora na capacidade aeróbica (Vo2max), força (isocinética e isométrica) e a capacidade funcional (Külkamp et al., 2012).

Dentre as mazelas apresentadas por pessoas acometidas com AR, 20-30\% têm redução da capacidade aeróbica e natural diminuição dos índices de força e massa muscular ocasionada pela dor, fadiga e limitação funcional das articulações em 
virtude da doença. Situações de risco cardiovascular podem ser apresentadas por pacientes de AR devido às limitações físicas e redução das práticas de exercícios (Silva, Costa, Oliveira, Muniz, \& Mota, 2013).

Com a finalidade de melhorar a capacidade cardiovascular e auxiliar na prevenção das limitações ocasionadas pela AR, algumas atividades aeróbicas são indicadas, por exemplo: Caminhar, correr, pedalar e praticar atividades aquáticas como natação e hidroginástica (Silva et al., 2013).

A prática regular de exercício ameniza o processo inflamatório, a dor e o acumulo anormal de líquidos nas articulações, auxiliando para restaurar a amplitude da mobilidade articular, força e aumento positivo do sistema cardiovascular (Gomes et al., 2013).

Indica-se para pessoas saudáveis, e inclusive para indivíduos com AR, que a prática de $\mathrm{EF}$ tenha duração mínima de 20 minutos, que seja efetuado ao menos duas vezes por semana e tenha um aumento de $60 \%$ dos batimentos cardíacos predita para a idade, objetivando apresentar efeitos clínicos satisfatórios e sem detrimento à doença, ou seja, sem o agrave de sua atividade e sem incidência de dores. Se comparar o exercício dinâmico ao processo de reabilitação articular convencional, nota-se uma significativa melhora da qualidade de vida dos pacientes com AR (Silva et al., 2013).

\section{Materiais e Métodos}

A pesquisa foi realizada através de um estudo de caso de caráter quantitativo e qualitativo com uma mulher adulta, de 49 anos de idade, diagnosticada com AR aguda, baixa mobilidade e até a data de término desta pesquisa encontrava-se acamada. A partir da criação de um protocolo de treinamento adaptado que almejasse desenvolver suas aptidões físicas e funcionais.

A pesquisa qualitativa tem seu pressuposto nos fenômenos humanos, que através da visão do pesquisador almeja verificar os processos e fenômenos sociais que cercam sua pesquisa e seu objeto de estudo, levando em consideração crenças, valores e representações sociais. Ainda de acordo com a autora, a pesquisa quantitativa se caracteriza pela preocupação em quantificar dados, através de estatística para comprovar se a pesquisa é válida ou não (Knechtel, 2014). Já o estudo de caso, se caracteriza em se aprofundar de forma intensa e exaustiva trabalhando com um ou poucos casos (Gil, 2007).

Este estudo foi dividido em três etapas: 1-coleta de dados através de avaliação física, questionário de capacidade funcional, depressão e qualidade de vida; 2-intervenção a partir da aplicação do protocolo de treinamento; 3-reavaliação dos testes para obtenção de resultados comparativos.

\subsection{Etapa 1}

Esta etapa consistiu na obtenção de dados e informações da capacidade funcional e estado atual da doença para que se pudesse elaborar uma intervenção de forma adequada. Além de fornecer dados do atual percentual de gordura corporal e medidas antropométricas.

Para esta etapa, foi utilizado os protocolos de 7 dobras cutâneas de JACSON \& POLLOCK, o questionário HAQ (Health Assessment Questionnaire) para avaliar a capacidade funcional, o módulo WHOQOL-BREF (The World Health Organization Quality Of Life), que é um questionário internacional de qualidade de vida desenvolvido pela OMS (Organização Mundial da Saúde), em uma versão simplificada, e o BECK-BDI um questionário que avalia a existência ou não de um estado depressivo.

Como método de quantificação de dados, durante os dias de treinamento, utilizamos um diário de campo, que foi aproveitado para relatar todo o processo ocorrido durante cada sessão logo após o seu termino. Nele foi descrito situações pela paciente, por sua cuidadora, funcionária doméstica e familiares, servindo principalmente como uma forma de controle diário 
para delinear situações como dores, mal-estar, fraqueza, melhora de execução, melhora na técnica, progressão de carga e de intensidade no treino.

Quanto ao procedimento de avaliação física, utilizamos o protocolo de 7 dobras cutâneas, fomentado por JACKSON \& POLLOCK em 1978 - tendo sua atualização por WARD em 1980 - no qual eles elaboraram equações para a estimativa de densidade corporal de homens e mulheres.

O método das dobras cutâneas é bastante utilizado para avaliar a composição corporal, tendo por objetivo prognosticar a gordura corporal relativa (GCR) e a massa gorda (MG), através de equações de regressão. As medidas foram realizadas em várias regiões do corpo para alcançar uma média da MG total. Essa repartição das dobras cutâneas se deve ao fato de que a distribuição do tecido adiposo não se apresenta da mesma forma e quantidade em todas as partes do corpo, havendo a obrigação de realizar medidas em locais diferentes para ter um resultado mais significativo (Machado, 2008).

Utilizou-se das seguintes dobras cutâneas: subescapular, que está localizada no sentido diagonal da escapula tendo por referência anatômica a borda medial da escápula e ângulo inferior da escápula; tricipital, situada no sentido vertical por referência anatômica o ponto meso-umeral; peitoral, que se encontra no sentido diagonal com referência na linha axilar anterior e mamilo; axilar média, disposta no sentido horizontal na junção entre xifoesternal e linha axilar media; supra ilíaca, posicionada no sentido diagonal ou obliquo com referência anatômica na crista ilíaca; abdominal, fixada no sentido horizontal ou vertical da cicatriz umbilical; e coxa, estabelecida na linha medial femoral com referência anatômica na borda superior da patela (Machado, 2008).

Para obtenção do resultado, primeiramente deve calcular a Densidade Corporal (DENS) para depois então utilizar a fórmula de Siri, chegando assim no percentual de gordura. É somado o número total do resultado das Dobras Cutâneas (DC) e colocado na fórmula DENS $=1,09700000-[0,00046971 *(\mathrm{DC})]+\left[0,00000056 *\left(\mathrm{DC}^{2}\right)\right]-[0,00012828 *($ idade $)]$. Formula Siri $\% \mathrm{G}=[(4,95 / \mathrm{DENS})-4,50] * 100$ (Margoti, 2009).

Para avaliar a capacidade funcional, empregamos o questionário HAQ (Health Assessment Questionnaire), validado na língua Portuguesa por (Ferraz, 1990). O HAQ avalia a gravidade da doença através de oito áreas das funções diárias de um paciente. O indivíduo descreve suas implicações ao exercer cada atividade em uma escala de zero a três, no qual o maior resultado de cada área é somado e sua média expõe o resultado total do HAQ. O escore pode ir de zero (Função Excelente) a três (Função Muito Ruim) (Amorim, 2011).

O módulo WHOQOL-BREF (The World Health Organization Quality Of Life) é um questionário internacional desenvolvido pela OMS (Organização Mundial da Saúde) para avaliar a qualidade de vida da população, em uma versão simplificada. Este questionário contém 26 questões, sendo as duas primeiras perguntas sobre a percepção de qualidade de vida e satisfação com a saúde. As outras 24 questões são divididas em 4 domínios: Físico, Psicológico, Relações Sociais e Meio Ambiente (World Health Organization Quality of Life Assessment, 1995).

No Domínio Físico (DF), pretende-se avaliar a dor e o desconforto, energia e fadiga, sono, repouso, mobilidade, atividades da vida cotidiana, dependência de medicações e tratamentos e capacidade do trabalho; o Domínio Psicológico (DPS) almeja aferir os sentimentos positivos, pensar, aprender, memória e concentração, autoestima, imagem corporal e aparência, sentimentos negativos, espiritualidade/ religião/crenças pessoais; com o Domínio de Relações Sociais (DS) deseja estimar relações pessoais, suporte social e atividade sexual; já o Domínio de Meio Ambiente (DA) anseia aquilatar segurança física e proteção, ambiente no lar, recursos financeiros, cuidados de saúde e sociais: disponibilidade e qualidade, oportunidades de adquirir novas informações e habilidades, participação e oportunidades de recreação/lazer, ambiente físico (poluição, ruído, trânsito, clima) e transporte.

Para obtenção do resultado de cada domínio é somado o número correspondente a cada resposta obtida e depois dividido pelo número de questões que compete cada domínio, ou seja, uma média do resultado total. A partir da média a 
condição do indivíduo é analisada da seguinte forma: 1 até 2,9 o paciente necessita melhorar, de 3 a 3,9 a QV é considerado regular, de 4 até 4,9 é considerado boa e 5 é considerado muito boa a QV.

Este questionário é como o indivíduo avaliado sente-se em relação a sua qualidade de vida, saúde e outros aspectos de sua vida, sem importar a avaliação ou julgamento de terceiros. O WHOQOL foi desenvolvido levando em consideração o motivo de que a compreensão de qualidade de vida é amplo e pode ser aposto a várias doenças e a casos não-médicos (Fleck, 2000).

Para coletar informações de anamnese do paciente, utilizamos de um instrumento de coleta de dados de pacientes adultos com AR, elaborado por Aline Rodrigues de Abreu Miranda, Carla Silva de Araújo, Fabiana Santana Faleiros-Castro no ano de 2012. A validação do conteúdo foi realizada por enfermeiros com experiência assistencial em AR e conhecimento da teoria (Miranda, Araújo, \& Faleiros-Castro, 2012).

Para obter dados que apontassem alguma incidência de depressão, foi aplicado o inventário de depressão BECK-DBI, constituído por 21 questões que foram respondidas tomando por base a última semana do indivíduo, devendo optar pelas alternativas das questões que melhor se enquadre, enumeradas de 0 a 3 , sendo zero o menor grau de incidência e três o maior grau de incidência na escala de depressão. Após a aplicação, os resultados serão somados e classificados de acordo com sua totalização, de 0 a 13 nenhuma depressão, de 14 a 19 depressão leve, de 20 a 28 depressão moderada, de 29 a 63 depressão grave.

\subsection{Etapa 2}

Após a coleta dos dados e avaliação dos questionários aplicados, esta etapa teve por finalidade intervir de forma direta efetuando EF com objetivo de melhora no que diz respeito à mobilidade articular, força, equilíbrio e percentual de gordura. Para isso, fizemos uma anamnese do paciente contendo dados pessoais, modo de interdependência, modo de autoconhecimento, modo fisiológico, seus sentidos, sua nutrição e sua atividade e repouso.

A intervenção aconteceu durante um período de três meses, definidos os dias de terça, quinta e sábado. O local de aplicação dos exercícios ocorreu dentro da casa da paciente, mais especificamente no quarto, devido à baixa mobilidade e grande dificuldade de locomoção para outro local.

A paciente locomove-se através de cadeira de rodas com ajuda de alguém, permanece a maior parte do tempo deitada ou sentada, isso dificulta os exercícios que exigem um pouco mais de dinamicidade. Os exercícios foram adaptados para que pudessem ser executados pela pessoa, deitada ou sentada, mas que consigam trabalhar os membros superiores e inferiores por completo. As atividades foram planejadas levando em consideração a funcionalidade do indivíduo, com a finalidade de observar melhoras em seu estado de capacidade funcional.

A intensidade de treinamento foi baixa, com a durabilidade de 45 minutos a 1 hora, composto por 6 a 8 exercícios aproximadamente e 3 séries em cada atividade, com o número de 10 repetições por série e o tempo de descanso de um minuto entre as series. A periodicidade de aumento de intensidade do treinamento ocorreu a cada duas semanas para que não tivesse uma adaptação completa dos estímulos utilizados.

Das intensidades de treinamento, utilizamos como forma de modificação de intensidade: o aumento da amplitude de movimento, diminuição da velocidade de movimento, menor tempo de descanso, exercícios de contração estática e o aumento da sobrecarga.

Os exercícios foram compostos por movimentos uniarticulares e multiarticulares. Abaixo está exposta na tabela 1 a lista de exercícios para membros superiores e inferiores utilizados na intervenção. Os materiais utilizados para execução dos exercícios foram compostos por fitas Thera-Band, bolas ortopédicas, elásticos, bolas cravo ante stress, halteres e anilhas. 
Tabela 1 - Exercícios para membros superiores e inferiores aplicados durante a intervenção

Exercícios de Membros Superiores

Exercícios de Membros Inferiores

Apertar A Bola

Elevação Lateral

Tríceps Testa

Rosca Direta

Exercício de Respiração Abdominal

Abdução Transversal De Ombro

Supino Vertical

Rosca Alternada
Abdução de Quadril

Adução de Quadril

Extensão de Joelho

Elevação da Pelve

Adução de Quadril

Flexão de Quadril

Exercícios de Membros Inferiores

Abdução de Quadril

Fonte: Autores.

\subsection{Etapa 3}

Esta etapa consistiu na reaplicação de todos os testes, questionários e análises feitas na primeira etapa. O objetivo foi coletar dados que comprovem a eficácia do protocolo de treinamento desenvolvido neste estudo e com isso analisar a melhora da paciente estudada.

Os dados coletados foram analisados e quantificados conforme as recomendações individuais de cada teste para comparação da autonomia funcional, percentual de gordura, nível de depressão e qualidade de vida antes da aplicação do protocolo de treinamento e após. Para isso utilizou-se como sistema de análise de dados o programa BioStat ${ }^{\circledR}$ 5.3.

A participante teve consentimento expressado através da assinatura ao Termo de Consentimento Livre e Esclarecido (TCLE), baseado na resolução no 510 , de 07 de abril de 2016.

\section{Análise Estatística}

Os dados obtidos foram analisados pelo programa de estatística BioStat ${ }^{\circledR}$ 5.3, nele calculamos a média, desvio padrão (DP), coeficiente de variação $(\mathrm{CV})$ e o percentual de mudança $(\Delta \%)$, os gráficos foram gerados através do programa Microsoft Excel 2010 com o intuito de ilustrar os escores obtidos.

\section{Resultados e Discussões}

\subsection{Anamnese do caso}

Dos Dados Pessoais, a paciente em estudo é mulher, com a idade de 49 anos, brasileira, solteira, com escolaridade de ensino técnico. Atualmente encontra-se aposentada, porém, trabalhava para o Governo Federal com a Saúde Indígena. Mora em casa alugada e possui renda adequada para a sua manutenção. Apesar disso, pudemos observar que sua locomoção para os demais cômodos da casa se dá através de cadeira de rodas, o que torna a casa inadequada para sua deslocação, por possuir vários níveis de degraus e nenhuma acessibilidade, com isso acaba passando a maior parte do tempo no quarto assistindo à televisão

No modo de Interdependência, existem algumas pessoas que mais ajudam no seu tratamento, que são a sua cuidadora, a mãe e a secretária doméstica. Relatou-se que o sujeito não tem dificuldades de interação familiar, porém sente muita solidão. 
Com isso, pudemos perceber que em alguns momentos ela acaba evitando contato com o meio exterior e evita também a relação com amigos. Durante seu processo de tratamento, sua cuidadora permanece em um período integral ao seu lado.

Na seção de Autoconceito, o diagnóstico médico principal é AR e em estado grave, pois se encontra acamada, porém consegue locomover-se com o auxílio de cadeira de rodas e de alguém empurrando, por não possuir força suficiente nos membros superiores. Foi-nos relatado que possui conhecimento do relatório médio e que tem muita expectativa de melhoras.

Relatou-nos não possuir nenhuma preocupação, bem como não ter vida sexual ativa e nem parceiro, pois houve o afastamento ao decorrer do agrave da doença. Os primeiros sintomas desta enfermidade surgiram por volta de 1996, durante os últimos meses os sintomas encontram-se estabilizados e sem nenhuma internação recente. Sua última internação foi em fevereiro de 2017, e sua última cirurgia foi em 10 de novembro de 2018, em Belém-PA.

Sobre medicamentos, sabe a indicação de todas as medicações e as utiliza corretamente. Seu acompanhamento médico deveria ocorrer na periodicidade de 3 em 3 meses, porém não vai ao médico devido ao percurso de deslocamento de Tucuruí à Belém, nos foi relatado por seus familiares um leque de desculpas inventadas para que não o faça. Além disso, deveria possuir acompanhamento Nutricional, Reumatológico, psicológico e Fisioterápico, entretanto, quando precisa sair de casa sempre chora e reluta para não ir.

É católica, contudo, não frequenta nenhuma congregação. Após o agrave da doença houve muitas alterações em relação ao seu estilo de vida, era uma pessoa completamente independente e hoje seu atual estado é de total dependência por encontrar-se acamada ou em uma cadeira de rodas. Não se sabe ao certo, mas devido a estes fatores pensamentos suicidas passaram por sua cabeça, porém relatou não ter coragem o suficiente para fazer. Sempre foi uma pessoa vaidosa e hoje não está satisfeita com sua aparência. Apesar disso, diz ser uma pessoa otimista e ao mesmo tempo irritada, tendo dias de altos e baixos. Contudo pudemos notar que em alguns momentos durante a aplicação do questionário, algumas informações foram ocultadas ou mascaradas, pois sua feição era sempre cabisbaixa.

No segmento Fisiológico e suas funções neurológicas, sua consciência é alerta, sabendo tudo que ocorre dentro de casa, sua atenção é íntegra, não houve alterações em sua memória e sua fala continua normal. Tem um comportamento combativo/agressivo, emocionalmente aparenta ser triste, deprimida e retraída. Não faz utilização de drogas ilícitas e nem cigarro, não tem alteração cardíaca, porém houve alterações respiratórias, com dificuldades de respirar e forte dor na região torácica, contudo foram tomadas apenas medidas paliativas, pois não teve nenhum diagnóstico concreto, além disso, é hipertensa e anêmica.

Em seus sentidos, a audição é normal, não utiliza aparelho auditivo, visão ilesa e não utiliza de lentes corretivas. Expôs-nos sentir dores muito fortes na região lombar e no joelho esquerdo, já no pé esquerdo é moderado, porém quando coloca o pé no chão a dor é intensa e constante, e não tem tempo estimado de duração. As formas de alívio se dão através de medicação, massagem ou aquecimento do local da dor.

Em sua Nutrição, não possui alterações gastrointestinais e nem diabetes, mas seu apetite sofreu alteração, pois não sente vontade de comer, são raros os dias que consegue fazer todas as refeições devidas, quando consegue realizar são apenas duas refeições por dia e com muito esforço. Para suprir sua necessidade de nutrientes faz uso de suplementos multivitamínicos e afirma ter uma boa ingestão de água, porém seus familiares relataram o contrário, que a paciente não faz utilização de água, e quando o faz, sente muita ânsia, e não ingere bebidas alcóolicas. Nos últimos 6 meses perdeu mais de 10 quilos, seu peso atual é de 43 quilos e sua altura de 1 metro e 52 centímetros.

Sobre Atividade e Repouso, nos foi relatado que as atividades de comer, aprontar-se, tomar banho, vestir parte inferior do corpo, ir ao banheiro, mobilidade, transferência, locomoção e escadas é de total dependência de sua cuidadora. Vestir parte superior do corpo ainda consegue, porém com alguma dificuldade, mas na verdade observamos que também tem total dependência neste quesito. Sua locomoção dar-se-á através de cadeira de rodas, porém passa a maior parte do tempo acamada. 
Não faz utensilio de órtese, possui deformidade em alguns membros e articulações do corpo, como por exemplo, nas articulações do calcanhar, joelho esquerdo, mãos direita e esquerda, e no cotovelo esquerdo. Não apresenta rigidez matinal e nenhum problema para dormir, porém isso só é possível desde que esteja medicada. No seu último emprego passava a maior parte do tempo em pé ou andando. Sua única pratica de lazer atualmente é assistir à televisão, nunca foi uma pessoa que gostou de práticas esportivas.

\subsection{Panorama geral dos resultados}

Pretende-se, nesta subseção, expor os dados gerais dos protocolos utilizados no estudo, tais como de Avaliação Física, Qualidade de vida, Capacidade Funcional (CF) e Depressão (DPR). A aplicação do treinamento teve a durabilidade de 14 semanas, com duas avaliações realizadas antes do início do programa (pré-teste) e ao término das aplicações (pós-teste), durante o período a paciente apresentou problemas relacionados à sua saúde mental, acarretando em algumas semanas de paralisação dos exercícios por opção dela, alegando dores ou indisposição.

A tabela 2 a seguir representa os resultados obtidos a partir da avaliação dos questionários e testes aplicados, trata-se de uma análise descritiva.

Tabela 2 - Resultados estatísticos referentes à avaliação física, qualidade de vida, capacidade funcional e depressão

\begin{tabular}{c|ccc|cccc}
\hline TESTE & - & PRÉ-TESTE & PÓS-TESTE & MÉDIA & DP & CV(\%) & $\Delta \%$ \\
\hline \multirow{2}{*}{ MAGRA } & 32.98 & 33.83 & 33.40 & 0.60 & 1.80 & $2,5 \%$ \\
& GORDA & 10.01 & 8.7 & 9.35 & 0.93 & 9.96 & $13.0 \%$ \\
& PERCENTUAL & 23.31 & 20.44 & 21.87 & 2.03 & 9.28 & $12,3 \%$ \\
& IMC & 18.61 & 19.14 & 18.87 & 0.37 & 1.99 & $2,8 \%$ \\
\hline \multirow{2}{*}{ CF } & DF & 3.14 & 2.14 & 2.64 & 0.70 & 26.78 & $31,8 \%$ \\
\hline DPR & DPS & 3 & 2.6 & 2.80 & 0.28 & 10.10 & $13,3 \%$ \\
& DS & 3.33 & 3.3 & 3.31 & 0.02 & 0.64 & $0,9 \%$ \\
& DA & 3.1 & 3.25 & 3.17 & 0.10 & 3.34 & $4,8 \%$ \\
\hline
\end{tabular}

Fonte: Autores.

Após a análise, os resultados obtidos mostram uma evolução boa em alguns aspectos, outros apresentam resultados tímidos ou negativos com base no protocolo individual de cada teste. Na avaliação física a paciente apresentou resultados satisfatórios quanto à massa magra $(33.40 \pm 0.60, \Delta \%=2,5 \%)$, massa gorda $(9.35 \pm 0.93, \Delta \%=13.0 \%)$, percentual de gordura $(21.87 \pm 2.03, \Delta \%=12,3 \%)$, IMC $(18.87 \pm 0.37, \Delta \%=2,8 \%)$.

$\mathrm{Na}$ análise da qualidade de vida, obtivemos os seguintes resultados em cada domínio: DF $(2.64 \pm 0.70, \Delta \%=31,8 \%)$, no DPS $(2.80 \pm 0.28, \Delta \%=13,3 \%)$, DS $(3.31 \pm 0.02, \Delta \%=0,9 \%)$ e no DA $(3.17 \pm 0.10, \Delta \%=4,8 \%)$. Os resultados obtidos na Capacidade Funcional (CF) foram singelos, porém significantes $(2.68 \pm 0.08, \Delta \%=4,7 \%)$. E por fim, no teste de depressão, a paciente apresentou melhora tímida no que tange aos resultados estatísticos $(22.50 \pm 2.10, \Delta \%=12,5 \%)$.

$\mathrm{O}$ coeficiente de variação mostra a partir dos dados se o resultado foi mais homogêneo ou discrepante, os números obtidos na análise desse estudo, em grande parte, nos mostram baixo grau de variação, que se encontram no resultado de massa 
magra $(\mathrm{CV} \%=1,80)$, gorda $(\mathrm{CV} \%=9,96)$, percentual $(\mathrm{CV} \%=9,28)$, IMC $(\mathrm{CV} \%=1,99)$, DS $(\mathrm{CV} \%=0,64), \mathrm{DA}(\mathrm{CV} \%=3,34)$, HAQ $(\mathrm{CV} \%=3,29)$ e BECK $(\mathrm{CV} \%=9,43)$; um teste gerou alterações no CV considerados como médio DPS $(\mathrm{CV} \%=10,10)$; e um gerou variação considerada de grau alto DF (CV\%26,78).

\subsection{Teste de capacidade funcional}

Como teste de autonomia funcional, o estudo objetivou aplica-lo em duas etapas: pré e pós-intervenção do protocolo de EF, tratando-se de um questionário de 20 questões que analisa 8 componentes, as questões foram respondidas pela própria paciente dentre as afirmativas que ela considerava mais semelhante à sua rotina.

Com base nos resultados obtidos pode-se notar uma pequena melhora nos componentes 2 e 5 , que correspondem a levantar-se e higiene pessoal respectivamente; nos componentes 3, 4, 6, 7 e 8 que correspondem a alimentar-se, caminhar, alcançar objetos, apreender objetos e outras atividades, as respostas obtidas foram iguais, ou seja, não havendo alterações; e no componente 1 que se refere a vestir-se, houve um declínio de um ponto em relação ao teste antes da intervenção. No pré-teste o resultado médio obtido foi de 2.75 , já no pós-teste obtivemos um valor numérico de 2.625 , comparando os resultados percebemos uma diferença de 0.125 que corresponde a uma leve melhora. A tabela 3 abaixo apresenta os valores alcançados no HAQ.

Tabela 3 - Resultados do HAQ

\begin{tabular}{|c|c|c|c|}
\hline Componentes & Perguntas & $\begin{array}{c}\text { Maior Score } \\
\text { Antes }\end{array}$ & $\begin{array}{c}\text { Maior Score } \\
\text { Depois }\end{array}$ \\
\hline Componente 1 (Vestir-se) & Perguntas 1 e 2 & 1 & 2 \\
\hline Componente 2 (Levantar-se) & Perguntas 3 e 4 & 3 & 2 \\
\hline Componente 3 (Alimentar-se) & Perguntas 5,6 e 7 & 3 & 3 \\
\hline Componente 4 (Caminhar) & Perguntas 8 e 9 & 3 & 3 \\
\hline Componente 5 (Higiene Pessoal) & Perguntas 10,11 e 12 & 3 & 2 \\
\hline Componente 6 (Alcançar Objetos) & Perguntas 13 e 14 & 3 & 3 \\
\hline Componente 7 (Apreender Objetos) & Perguntas 15,16 e 17 & 3 & 3 \\
\hline \multirow[t]{3}{*}{ Componente 8 (Outras Atividades) } & Perguntas 18,19 e 20 & 3 & 3 \\
\hline & Soma Total $=$ & 22 & 21 \\
\hline & Resultado $=$ & 2.75 & 2.625 \\
\hline
\end{tabular}

Fonte: Autores.

Autores que utilizaram do memo método em sua pesquisa identificaram em seus estudos a melhora da capacidade funcional de 53 indivíduos acometidos com AR, obtendo uma média de 1,53 no teste HAQ, o período de análise foi de 7 meses (Corbacho \& Dapueto, 2010).

Já outro grupo de pesquisadores efetuou uma análise prospectiva de 3 anos com indivíduos diagnosticados com AR no estado inicial da doença, no total foram 40 pessoas, chegando a uma média de 1,8 na primeira avaliação e 0,77 após três anos (Mota, Laurindo, \& Santos Neto, 2010b).

As investigações acima corroboram com os resultados de nossa pesquisa na melhora da capacidade funcional, expressando números que comprovam a evolução após intervenção por meio de EF, compreendendo que estes estudos se 
tratam com um número de pessoas maior em relação ao nosso e que apresentam níveis de atividade da doença inferiores à nossa pesquisa, tornando difícil a comparação de resultados.

\subsection{Teste de qualidade de vida}

Para analisar a qualidade de vida, aplicamos o questionário internacional de qualidade de vida fomentado pela OMS, em uma versão simplificada que contém 26 questões e divide em 4 domínios: Físico, Psicológico, Social e Ambiental. Os resultados estão expostos no gráfico 1 abaixo:

Gráfico 1 - Resultados do teste de qualidade de vida

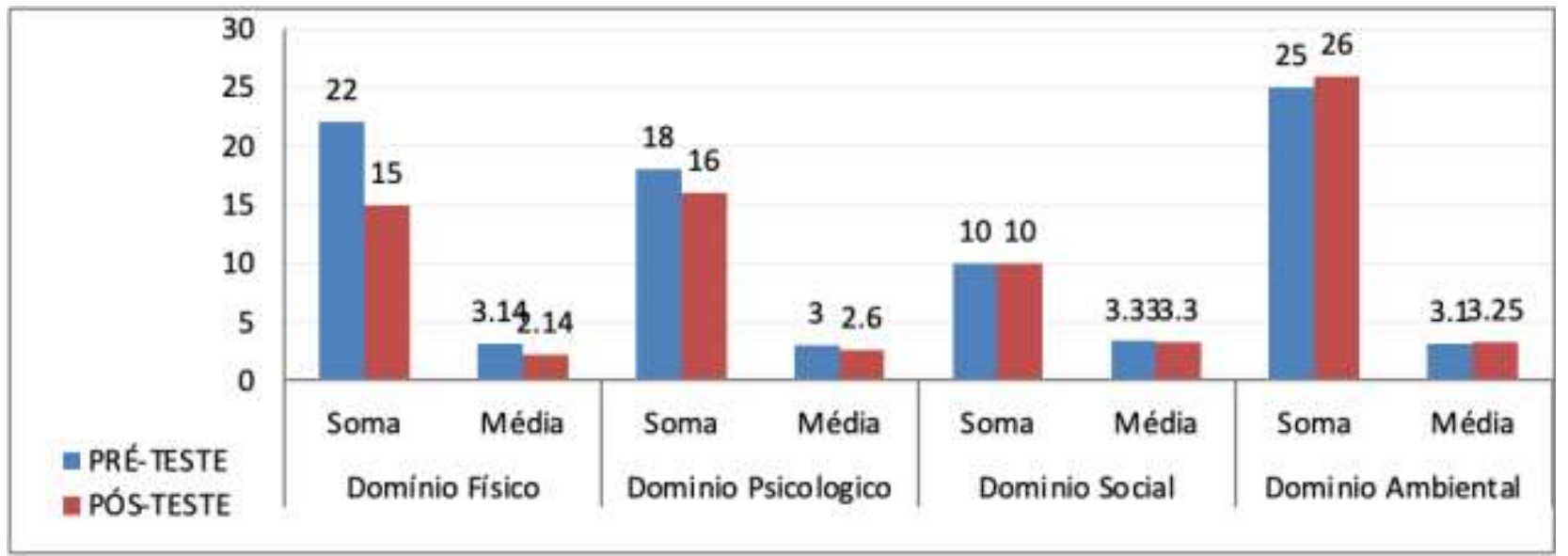

Fonte: Autores.

A partir dos resultados obtidos e seguindo a referência da OMS na qual classifica o score até 2.9 como necessita melhorar, de 3.0 a 3.9 como regular, de 4.0 a 4.9 como boa e 5 como muito boa. No pré-teste, em relação ao Domínio Físico, obtivemos uma soma total de 22 pontos, sua média foi 3,14 e isso classifica a média como regular; no Domínio Psicológico a soma total obtida foi de 18 pontos, sua média foi de 3, também classificado com regular; no Domínio Social a soma total foi de 10 pontos na média 3,33 considerado com regular; e no Domínio Ambiental a soma total foi de 25 e a média foi 3,125, estimado com regular. Todos os domínios foram classificados como regular no primeiro teste.

Já no pós-teste, nos resultados obtidos foram: no Domínio Físico a soma total foi de 15 e a média 2,14, que de acordo com a tabela base se classifica como necessita melhorar; no Domínio Psicológico a soma total foi 16 e a média 2,66, também considerada como necessita melhorar; no Domínio Social a soma total foi de 10 e a média 3,33, classificada como regular; e no Domínio Ambiental a soma total foi 26 e a média 3,25, considerado regular. Dos 4 domínios 2 foram classificados como necessita melhorar e 2 como regular.

Fazendo a comparação entre os resultados de cada domínio podemos observar que houve uma queda nos Domínios Físico e Psicológico no pós-teste. Este decréscimo em relação ao resultado pode estar associado a interferência de doenças psicossomáticas durante o período de intervenção e ao aumento do processo inflamatório relatado pela paciente, acarretando na interrupção do treinamento em um período de 1 mês. Um resultado semelhante foi apresentado em um estudo publicado por outros autores, cujo os domínios de relações sociais e intimidades não houveram alterações (Soares, Oliveira, Silva, \& Barbosa, 2017).

Efeitos parecidos apresentados em um estudo mostraram a interferência da Velocidade de Hemossedimentação (VHS) na qualidade de vida dos pacientes estudados; o VHS se ligou inversamente com o Domínio Físico e Psicológico, o que indica 
que a atividade da doença pactuou na qualidade de vida, ou seja, com o avanço do processo inflamatório, os pacientes apresentaram escores menores em todos os domínios do WHOQOL-BREF (Prates, 2012).

\subsection{Escala de depressão beck}

Questionário aplicado para analisar o nível de depressão que a paciente apresentava antes e depois da nossa intervenção, o teste é constituído por 21 questões em que soma das respostas gera o número total, o teste classifica o total obtido em: nenhuma depressão, depressão leve, depressão moderada e depressão grave. Os resultados adquiridos estão expressos no Gráfico 2.

Gráfico 2 - Beck de depressão

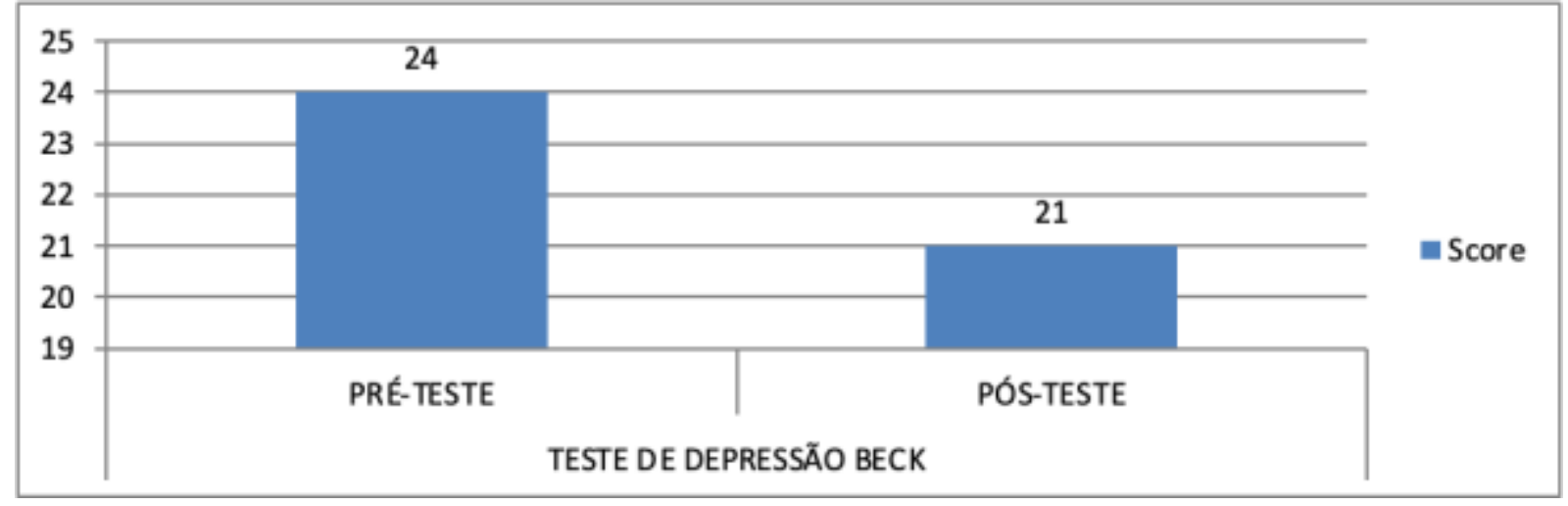

Fonte: Autores.

No primeiro pré-teste obtivemos a soma total de 24 pontos classificada como depressão moderada e no pós-teste a soma obtida foi de 21 pontos também classificada como moderada, porém com uma queda de 3 pontos.

Em um estudo similar algumas autoras analisaram 60 indivíduos divididos em dois grupos, um com 30 pessoas diagnosticadas com AR e outro com 30 pessoas sem complicações médicas, chegou ao resultado alarmante, no qual o grupo com AR apresentou 63,33\% (classificado com algum grau de depressão) contra 13,34\% do grupo sem complicações médicas (Campos, Silva, Castro, \& Graminha, 2013).

\subsection{Avaliação física}

O gráfico 3 diz respeito aos valores do Percentual de Gordura (G\%), Massa Magra (MM), Massa Gorda (MG) e Índice de Massa Corporal (IMC) do pré-teste e pós-teste. Do G\% o valor obtido no pré-teste foi de 23,31\% e no pós-teste foi de $20,44 \%$, assim, obtivemos uma variação positiva de $2,87 \%$ entre os resultados. A quantidade de MM no pré-teste foi de $32,98 \mathrm{~kg}$ e pós-teste foi de 33,83 kg, com um aumento de 0,85 kg. Em relação a MG, o valor do pré-teste foi de $10.02 \mathrm{~kg}$ e pósteste de $8,70 \mathrm{~kg}$, obtendo uma redução de 1,32 kg. No IMC o valor do pré-teste foi de 18,61 e do pós-teste de 19,14, apresentando um aumento de 0,53 entre os testes. 
Gráfico 3 - Resultados da avaliação física

\begin{tabular}{|c|c|c|c|c|}
\hline \multicolumn{4}{|c|}{32.9833 .83} \\
\hline
\end{tabular}

Fonte: Autores.

Fazendo uma análise minuciosa dos dados obtidos, podemos observar que houve resultados positivos em todos os quesitos. Apesar de ter uma diminuição do peso total, houve uma redução da MG e um aumento da MM, obtendo assim, um resultado satisfatório na diminuição da quantidade total de tecido adiposo que compunha o corpo da pessoa avaliada. Outro fator importante foi o aumento do IMC, apesar de que a faixa classificatória continua a mesma (peso normal), no pré-teste a estudada estava próximo do estado de abaixo do peso, o que significaria uma má nutrição do seu corpo.

Os estudos de alguns autores apontam resultados contrários aos que obtivemos, em que a presença do sobrepeso é mais comumente observada em pacientes com AR. Entretanto, um baixo nível de G\% pode reduzir o processo inflamatório, pois, o tecido adiposo não é apenas uma fonte reserva de energia, ele também secreta moléculas de adipocinas, nas quais altos níveis de adipocinas estão associados ao aumento do processo inflamatório, tornando a obesidade reconhecida atualmente como um estado pré-inflamatório (Armstrong, McCausland, Quinn, \& Wright, 2006; Naranjo et al., 2008; Zarpellon, Dias, \& Skare, 2014).

\section{Conclusão}

Após análise dos testes aplicados, podemos deduzir que os exercícios físicos têm um impacto significativo no que se refere ao tratamento de AR. Dos 4 testes aplicados, conseguimos observar uma melhora no Beck DBI, Capacidade Funcional e 7 Dobras de Jack e Pollock. No Teste de qualidade de vida tivemos um déficit em relação aos domínios físicos e psicológicos. Contudo, este déficit pode ter relação com a interrupção da aplicabilidade do protocolo de treinamento através do desencadeamento de quadros depressivos, causando a interrupção das atividades, apresentando crises de choro, desânimo, enjoo e dores fortes nas articulações e na região torácica.

Portanto, a aplicação de exercícios físicos como um meio não medicamentoso no tratamento da AR para o indivíduo investigado se mostrou uma alternativa viável, que deve ser encarado como essencial no desenvolvimento do sujeito. Apesar disso, alertamos para a preocupação de doenças que podem ser desencadeadas com a AR, como depressão, ansiedade e outras doenças psicossomáticas que refletem no desempenho corporal dos pacientes, reforçando a necessidade de acompanhamento de uma equipe multiprofissional.

Conclui-se que o programa de treinamento para este estudo se mostrou eficaz. Todavia, sugere-se que a reaplicação dos testes que comprovem a eficácia do treinamento seja refeita em periodicidade mensal para que se obtenha um controle maior sobre os resultados, evitando situações adversas que podem ocorrer ao longo do tratamento; novos estudos devem ser realizados com um número maior de pessoas e com níveis dos estágios diferentes da doença, para que se consolidem os resultados encontrados neste estudo. 


\section{Referências}

Amorim, K. S. D. (2011). Relação entre o nível de atividade física e a concentração plasmática de homocisteína em mulheres com artrite reumatóide. Retrieved from http://repositorio.ufsc.br/xmlui/handle/123456789/95714.

Armstrong, D. J., McCausland, E. M., Quinn, A. D., \& Wright, G. D. (2006). Obesity and cardiovascular risk factors in rheumatoid arthritis. Rheumatology (Oxford), 45(6), 782; author reply 782-783. doi:10.1093/rheumatology/kel124.

Campos, A. P. R., Silva, C. M., Castro, S. S. d., \& Graminha, C. V. (2013). Depressão e qualidade de vida em indivíduos com artrite reumatoide e indivíduos com saúde estável: um estudo comparativo. [Depression and quality of life in rheumatoid arthritis individuals and stable health individuals: a comparative study

Depresión y calidad de vida en individuos con artritis reumatoidea e individuos con salud estable: un estudio comparativo]. Fisioterapia e Pesquisa, 20(4), 401-407.

Cavanaugh, S. V. A., Furlanetto, L. M., Creech, S. D., \& Powell, L. H. (2001). Medical illness, past depression, and present depression: a predictive triad for in-hospital mortality. Am J Psychiatry, 158(1), 43-48. doi:10.1176/appi.ajp.158.1.43.

Corbacho, M. I., \& Dapueto, J. J. (2010). Avaliação da capacidade funcional e da qualidade de vida de pacientes com artrite reumatoide. [Assessing the functional status and quality of life of patients with rheumatoid arthritis]. Revista Brasileira de Reumatologia, 50(1), 31-43.

Costa, A. F. C. d., Brasil, M. A. A., Papi, J. A., \& Azevedo, M. N. L. d. (2008). Depressão, ansiedade e atividade de doença na artrite reumatóide. [Depression, anxiety and disease activity in rheumatoid arthritis]. Revista Brasileira de Reumatologia, 48(1), 7-11. doi:10.1590/S0482-50042008000100003.

Ferraz, M. B. (1990). Tradução para o português e validação do questionário para avaliar a capacidade funcional "stanford health assessment questionnaire". (tese de doutorado), Escola Paulista de Medicina, Sao Paulo.

Fleck, M. P. d. A. (2000). O instrumento de avaliação de qualidade de vida da Organização Mundial da Saúde (WHOQOL-100): características e perspectivas. [The World Health Organization instrument to evaluate quality of life (WHOQOL-100): characteristics and perspectives]. Ciência \&amp; Saúde Coletiva, $5(1), 33-38$

Gil, A. C. (2007). Métodos e Técnicas de Pesquisa Social (Vol. 5). São Paulo: Atlas.

Gomes, R. P., Bressan, E., Silva, T. M. D., Gevaerd, M. D. S., Tonussi, C. R., \& Domenech, S. C. (2013). Padronização de modelo experimental adequado a estudos do efeito do exercício na artrite. [Standardization of an experimental model suitable for studies on the effect of exercise on arthritis]. Einstein (São Paulo), 11(1), 76-82.

Gualano, B., Pinto, A. L. D. S., Perondi, M. B., Roschel, H., Sallum, A. M. E., Hayashi, A. P. T., . . Silva, C. A. (2011). Efeitos terapêuticos do treinamento físico em pacientes com doenças reumatológicas pediátricas. [Therapeutic effects of exercise training in patients with pediatric rheumatic diseases]. Revista Brasileira de Reumatologia, 51(5), 490-496.

Knechtel, M. d. R. (2014). Metodologia da pesquisa em educação: uma abordagem teórico-prática dialogada (Vol. 1). Curitiba: Intersaberes.

Külkamp, W., Dario, A. B., Gevaerd, M. d. S., \& Domenech, S. C. (2012). Artrite reumatóide e exercício físico: resgate histórico e cenário atual. Revista Brasileira de Atividade Física \&amp; Saúde, 14(1), 55-64. doi:10.12820/rbafs.v.14n1p55-64

Laurindo, I. M. M., Pinheiro, G. d. R. C., Ximenes, A. C., Xavier, R. M., Giorgi, R. D. N., Ciconelli, R. M., . . Alencar, P. (2002). Consenso brasileiro para diagnóstico e tratamento da artrite reumatóide. [Brazilian consensus for the diagnosis and treatment of rheumatoid arthritis]. Rev. bras. reumatol, 42(6), 355361.

Louzada-Junior, P., Souza, B. D. B., Toledo, R. A., \& Ciconelli, R. M. (2007). Análise descritiva das características demográficas e clínicas de pacientes com artrite reumatóide no estado de São Paulo, Brasil. [Descriptive analysis of the demographical and clinical characteristics of the patients with rheumatoid arthritis in the State of São Paulo, Brazil]. Rev. bras. reumatol, 47(2), 84-90.

Machado, A. F. (2008). Dobras cutâneas: localização e procedimentos. Motricidade, 4(2), 42-46.

Margoti, T. (2009). Comparação de resultado entre as equações de composição corporal de jackson \& pollock de três e sete dobras cutâneas. Fitness \& Performance Journal, 8(3), 191-198.

Miranda, A. R. d. A., Araújo, C. S. d., \& Faleiros-Castro, F. S. (2012). Instrumento para coleta de dados de pacientes adultos com artrite reumatoide. [Instrumento para recolección de datos de pacientes adultos con artritis reumatoide Instrument for data collect of adult patients with rheumatoid arthritis]. Rev. enferm. Cent.-Oeste Min, 2(2), 228-242.

Mota, L. M. H. D., Cruz, B. A., Brenol, C. V., Pereira, I. A., Fronza, L. S. R., Bertolo, M. B., . . Pinheiro, G. D. R. C. (2011). Consenso da Sociedade Brasileira de Reumatologia 2011 para o diagnóstico e avaliação inicial da artrite reumatoide. [2011 Consensus of the Brazilian Society of Rheumatology for diagnosis and early assessment of rheumatoid arthritis]. Revista Brasileira de Reumatologia, 51(3), 207-219.

Mota, L. M. H. d., Laurindo, I. M. M., \& Santos Neto, L. L. d. (2010a). Artrite reumatoide inicial: conceitos. [Early rheumatoid arthritis: concepts]. Revista da Associação Médica Brasileira, 56(2), 227-229.

Mota, L. M. H. D., Laurindo, I. M. M., \& Santos Neto, L. L. D. (2010b). Avaliação prospectiva da qualidade de vida em uma coorte de pacientes com artrite reumatoide inicial. [Prospective evaluation of the quality of life in a cohort of patients with early rheumatoid arthritis]. Revista Brasileira de Reumatologia, 50(3), 249-261. doi:10.1590/S0482-50042010000300005

Naranjo, A., Sokka, T., Descalzo, M. A., Calvo-Alén, J., Hørslev-Petersen, K., Luukkainen, R. K., . . Pincus, T. (2008). Cardiovascular disease in patients with rheumatoid arthritis: results from the QUEST-RA study. Arthritis Res Ther, 10(2), R30. doi:10.1186/ar2383 
Research, Society and Development, v. 10, n. 9, e59410918481, 2021

(CC BY 4.0) | ISSN 2525-3409 | DOI: http://dx.doi.org/10.33448/rsd-v10i9.18481

Oliveira, A. V. d. (2015). Análise dos gastos do Ministério da Saúde com medicamentos para tratamento da artrite reumatoide no Brasil no período 2010 a 2014. Brasilia.

Prates, W. B. (2012). Perfil epidemiológico e qualidade de vida dos pacientes com artrite reumatóide em tratamento em um hospital de referência no Espírito Santo. Retrieved from http://repositorio.ufes.br/handle/10/5479,

Rosa, L. M. d., Schoëller, S. D., Menegon, F. H. A., Santos, A. L. d., Salum, N. C., \& Miranda, G. M. (2018). Modalidades e Benefícios da Atividade Física na Artrite Reumatóide: Estudo de Revisão. CIAIQ2018, 2, 10.

Silva, C. R., Costa, T. F., Oliveira, T. T. V. d., Muniz, L. F., \& Mota, L. M. H. dD (2013). Prática de atividade física entre pacientes da Coorte Brasília de artrite reumatoide inicial. [Physical activity among patients from the Brasília cohort of early rheumatoid arthritis]. Revista Brasileira de Reumatologia, 53(5), 394-399.

Soares, S. P., Oliveira, D. V. D., Silva, P. N. D., \& Barbosa, C. P. (2017). A influência dos exercícios funcionais na evolução da artrite reumatoide: um estudo de caso. 53(2).

Sociedade Brasileira de Reumatologia. (2017). Artrite Reumatoide Doença inflamatória crônica que pode afetar várias articulações e com causa ainda desconhecida. Retrieved from https://www.reumatologia.org.br/doencas-reumaticas/artrite-reumatoide/

World Health Organization Quality of Life Assessment. (1995). position paper from the World Health Organization. Soc Sci Med, 41(10), 1403-1409. doi:10.1016/0277-9536(95)00112-k

Zarpellon, R. S. M., Dias, M. M., \& Skare, T. L. (2014). Perfil nutricional na artrite reumatoide. [Nutritional profile in rheumatoid arthritis]. Revista Brasileira de Reumatologia, 54(1), 68-72. doi:10.1016/j.rbr.2014.01.007 